\title{
Práctica deportiva, alimentación y construcción del cuerpo
}

ELENA ESPEITX

Universidad de Zaragoza. Huesca

\section{RESUMEN}

El presente artículo pretende abordar la relación existente entre actividad físico-deportiva y alimentación en adolescentes y jóvenes. A través de estas líneas, se pretende revisar cómo, y en qué medida, el concepto de "construcción del cuerpo" interviene en esta relación, y especialmente, cómo se relacionan social y culturalmente la alimentación, el ejercicio y el deporte.

Palabras clave: Deporte, Alimentación, Ejercicio, Construcción del cuerpo.

\section{SUMMARY}

The author discusses the relationship between sporting physical activity and the nutrition of both youngsters and adolescents. She focuses on the way in which - and the extent to which - the concept of "construction of the body" partakes in this social and cultural relationship involving sports, nutrition and exercise.

Key Words: Sports, Nutrition, Exercise, Construction of the Body.

\section{INTRODUCCIÓN}

En este artículo se aborda la relación existente entre práctica deportiva y alimentación, en adolescentes y jóvenes. Se pretende ver cómo, y en qué medida, el concepto de "construcción del cuerpo" interviene en esta relación. ¿Cómo se relacionan alimentación y práctica deportiva? Por un lado, la práctica deportiva regular se traduce en muchos casos en comportamientos alimentarios específicos y supone también a menudo la adquisición de conocimientos nutricionales y sobre "dietas aconsejables", que favorezcan el rendimiento deportivo. Por el otro, las representaciones sobre imagen corporal, actividad física y alimentación no sólo aparecen como las principales vías de intervención sobre el cuerpo, sino que se perciben estrechamente 
imbricadas entre sí. Y ¿por qué centrarse en adolescentes y jóvenes? La adolescencia resulta particularmente interesante, puesto que es un período de cambios por lo que respecta a la práctica deportiva y el comportamiento alimentario, en el que se abandonan o se modifican las prácticas hasta el momento impuestas, o como mínimo dirigidas, por padres y educadores, y en el que empiezan a aparecer nuevas prácticas, y nuevas formas de pensar la alimentación, la actividad física y el deporte. Analizar los cambios en relación a la infancia, por lo que respecta a la actividad deportiva y la alimentación, y cómo estos cambios se racionalizan y se explicitan, nos permite ver de cerca cómo se va reinterpretando la construcción del cuerpo. Se ha señalado a menudo que la adolescencia es una etapa en la que se reduce el peso de los hábitos familiares, mientras que el grupo de amigos y las referencias sociales se convierten en condicionantes claves en la estructuración de la alimentación. La actividad física y el deporte, en tanto que ámbitos de relación social, de generación de redes y de transmisión de conocimientos y de modelos de comportamiento, pueden incidir en el sentido de una auténtica transformación en los estilos de vida de los adolescentes.

Asimismo, es preciso destacar que la preocupación por la imagen corporal es uno de los factores que incide — con mayor o menor fuerza- tanto en los comportamientos alimentarios como en la práctica deportiva, y esta doble influencia e interacción merece ser tomada en consideración. Por otro lado, ver cómo los jóvenes interpretan la relación entre actividad física, alimentación y práctica deportiva proporciona claves muy útiles, ya que la mayor parte de ellos toman decisiones marcadamente autónomas sobre estas cuestiones.

\section{SENTIDOS Y "FunCIONES" DE LA CONSTRUCCIÓN DEL CUERPO}

Comer no consiste únicamente en satisfacer las necesidades nutricionales, sino que, como toda actividad humana, adquiere un gran número de sentidos y de "funciones", que resultan, no del hecho individual de nutrirse, sino de la interacción de los individuos en sociedad, y de los sistemas de clasificación, normativos, prácticas e instituciones, que surgen de esta interacción y que al mismo tiempo la estructuran. Las relaciones, múltiples y complejas, entre alimentación y "construcción del cuerpo" son generadoras de buen número de estas "funciones" y de estos sentidos que se otorgan al hecho de comer.

Pero, ¿qué entendemos por "construcción del cuerpo"? Es sabido que aquello que ingerimos modela objetivamente nuestro cuerpo al aportar los nutrientes necesarios para que el organismo lleve a cabo sus funciones 
básicas. A partir de esta dimensión fundamental de la alimentación se despliega la dimensión simbólica de la "construcción del cuerpo" a través de la ingesta de alimentos. Veamos en qué consiste.

El impulso y la capacidad del ser humano de incidir sobre el entorno, sobre los demás, sobre sí mismo, se manifiesta constantemente y por distintas vías. A este impulso y capacidad de actuar se le añade el impulso — capacidad, necesidad - de elaborar sistemas de significados alrededor de esta actuación, y la capacidad —necesidad- de comunicarlos, de expresarlos. Una de las formas que adopta la actuación del ser humano sobre sí mismo consiste en "construir" el propio cuerpo. Construirlo por un lado objetivamente, físicamente, modelándolo, y construirlo por otro lado - y al mismo tiempo- conceptualmente, dando sentidos y "funciones" al resultado de este modelado, la imagen corporal. A través de esta creación, se actúa sobre tres dimensiones relevantes: a) la identidad, o el nosotros con, frente y para los otros, b) la autopercepción, o el nosotros para nosotros, y c) el atractivo sexual. Así, la construcción del cuerpo, y su resultado, la imagen corporal, se explican, en parte, porque el cuerpo es generador y portador de identidad. Alargar partes del cuerpo, comprimirlas, tatuar la piel, perforarla, escarificarla, pintarla, son comportamientos extendidos en las sociedades humanas, a lo largo del tiempo y a lo ancho de las culturas. Con esto, los seres humanos expresan poder, autoridad o sumisión, diferenciación o pertenencia, creencias y valores... En cualquier caso, pretenden manifestar quiénes son, qué roles se les atribuye, qué lugar ocupan en la sociedad. Así, a través de aquello que llamamos "la imagen corporal" los individuos muestran quiénes son —o quiénes quieren hacer creer que son- cómo son - o cómo quieren parecer que son-qué lugar ocupan en la sociedad - o que lugar quieren ocupar- y lo hacen de diferentes maneras, utilizando diferentes vías. Pero esta identidad proyectada, este nosotros para los otros, no agota las "funciones" y sentidos de la imagen corporal construida. También afecta - y puede hacerlo con fuerza- la autopercepción, la imagen que de uno mismo se devuelve el individuo, el nosotros para nosotros. Efectivamente, la construcción del cuerpo y la definición de la imagen corporal es social, o, más concretamente, psicosocial. Las relaciones que los individuos mantienen con su propio cuerpo son complejas, y a menudo conflictivas. Dependen de diversos factores: del estado de ánimo, de la intensidad de sus percepciones corporales y de sus percepciones sensoriales en un determinado momento, de la imagen que de sí mismos envían los otros, de la valoración, o al contrario, el rechazo que sienta respecto al propio cuerpo. La autopercepción del cuerpo no depende únicamente de las propias sensaciones corporales. Según si se rechaza o se aprecia —es decir, si encaja o no, o lo hace mejor o peor, con la proyección de la iden- 
tidad deseada - se percibe de manera distinta. Además, esta imagen subjetiva es inestable y no siempre - a menudo no lo hace- se corresponde con la realidad corporal. La imagen del cuerpo no es una construcción esencialmente interna, sino que es en gran medida tributaria de cómo lo perciben los otros, así como de la imagen de uno mismo que éstos transmiten. Así, en un doble movimiento, los individuos proyectan su identidad instrumental - la que les sirve para interactuar con los otros- a través de la imagen corporal y, a su vez, mediante la imagen corporal de sí mismos que les devuelven los otros, labran su autopercepción. Además de la identidad mostrada y de la identidad autopercibida, también a través de la imagen corporal se transmite el atractivo sexual, y esta no es, sin duda, una función que satisfaga una "necesidad" menor.

Como se ha señalado anteriormente, el lenguaje de la imagen corporal habla a lo largo del tiempo y a lo ancho de diferentes culturas y sociedades, pero lo hace en idiomas distintos y con significados y énfasis diferentes. En nuestras sociedades, habla con una voz muy fuerte e insistente. Y lo hace por diferentes motivos. Uno de ellos, que se ha apuntado a menudo, sería el individualismo imperante. Así, en una sociedad marcada por el individualismo como valor, como creencia y sistema de vida, la imagen corporal adquiere una gran relevancia, al convertirse en carta de presentación de los individuos, que se promocionan desde su individualidad. Desde esta perspectiva se considera que la persona, la presentación externa que hace de sí misma, siempre está mediada por su aspecto, de modo que su imagen se convierte en ella misma, hasta el punto de que sólo mediante un conocimiento muy próximo y continuado de esta persona resulta posible hacer abstracción de su imagen corporal (Alberdi 2000: 21). Ésta sería la razón por la que la imagen adquiriría una importancia destacada en la actualidad. Es cierto que los valores de individualización y de autonomía personal parecen presentes en nuestra sociedad, y que contribuyen al incremento del énfasis puesto en la imagen corporal, pero hay algunas dimensiones objetivas de la vida social que propician este incremento con aparente gran eficacia: a) multiplicación de los contactos breves con otros individuos, en el ámbito de las relaciones personales, b) aumento de la diversidad de situaciones - sucesivas y paralelas- en las que se encuentran los individuos, con la consiguiente movilidad en los roles, c) relaciones afectivas y sexuales no prefijadas, indeterminadas, que implican elecciones.

La multiplicación de contactos breves convierte la imagen corporal en la principal fuente de información sobre los individuos, y la mejor vía para una primera definición. Aunque todo esto es cierto, también aparecen nuevas formas de relación social que se desligan de la imagen corporal, porque esta desaparece, como aquellas relacionadas con Internet, que adquieren 
una importancia destacada, pero no sustituyen, en cualquier caso, las relaciones personales alimentadas por la información que proporciona la imagen corporal. Debido al aumento de la diversidad de contextos en los que se encuentran los individuos, en función de las diferentes situaciones y momentos de la vida, la imagen corporal puede modificarse y adaptarse a estos contextos cambiantes; a menudo debe hacerlo para resultar eficiente. Eficiente en la transmisión de información, en la comunicación. Un ejemplo obvio es que no se viste del mismo modo para ir a la playa que para ejercer de director de banco. Por lo que respecta a las relaciones afectivas y sexuales, en nuestra sociedad éstas no están predeterminadas, fijadas de antemano. Es decir, no son los padres u otros familiares quienes pactan el matrimonio del hijo, no hay rígidas leyes que decidan quiénes pueden ser cónyuges o no, ni la elección de pareja suele ser única y para siempre. Se hacen elecciones múltiples y sucesivas, y en la imagen corporal, el cuerpo construido, juega un papel como mínimo relevante.

No menos importante, más bien todo lo contrario, es que el negocio de la imagen corporal mueva cantidades ingentes de dinero y de intereses. Este no es un hecho banal, explica en buena medida algunas de las formas - sobredimensionadas- que adopta en nuestra sociedad esta tendencia humana a comunicar a través de la construcción del cuerpo y a instrumentalizar la imagen corporal.

\section{LA ALIMENTACIÓN EN LA CONSTRUCCIÓN DEL CUERPO}

Uno de los instrumentos que permiten esta construcción - entre muchos otros, como el vestido, el uso de abalorios y de símbolos de estatus diversos- es la alimentación. La relación entre alimentación e imagen corporal se percibe empíricamente estrecha, por ello la idea de que el ser humano puede actuar sobre la imagen corporal —es capaz de "construir su cuerpo" mediante lo que come- se encuentra extendida. La modelación del cuerpo mediante la alimentación y la expresión de la identidad y del atractivo sexual a través de la imagen corporal se manifiestan por dos vías fundamentales: la de ensanchar el cuerpo, mediante los consumos excesivos u ostentosos, y la de encoger, reducir el cuerpo, mediante la renuncia y la privación. Así, en un extremo, el objetivo consiste en agrandar el cuerpo, cubrirlo con capas de grasa que incrementen su volumen, lo que ha sido una práctica extendida a lo largo del tiempo y en diferentes sociedades para exhibir, a través de la imagen corporal, la abundancia de los propios recursos $^{1}$. En el otro extremo, encontramos la privación alimentaria autoim-

${ }^{1}$ Por ejemplo, entre los Jaina, era habitual — aún lo es, aunque en menor medidaque las suegras cebaran a sus nueras, para mostrar así, por un lado, la opulencia de la 
puesta. En la experiencia histórica de la humanidad existen abundantes ejemplos de ayuno voluntario y de restricción alimentaria severa, relacionados con diferentes sistemas de creencias y formas de pensar y de mostrarse al mundo ${ }^{2}$.

¿Qué sucede en nuestra sociedad? La vía para expresar poder o riqueza mediante el agrandamiento del cuerpo no parece, hoy por hoy, muy vigente, sin embargo la imposición de restricción alimentaria, con el objetivo de mantener dentro de unos límites — estrechos- los contornos de éste, lo es plenamente. Otra vía muy presente es la que consiste en desarrollar los músculos, como se verá más adelante, a través de ejercicios específicos, pero también con la ayuda del régimen alimentario. Optar por una u otra vía —adelgazar o muscular - está correlacionado significativamente con el género, lo cual no es banal.

Ya se ha dicho que el interés por la construcción del cuerpo, y su resultado, la imagen corporal, no es exclusivo de las sociedades actuales, pero sí es específica la forma que adopta esta construcción y los sentidos que se otorgan a la imagen resultante. Lo más significativo de la actual forma, particular y contingente, de construir el cuerpo y de interpretar la imagen corporal probablemente reside en la valoración de la delgadez en las mujeres. En los hombres esta imagen pasa en mayor medida por la musculación -elude por tanto la esbeltez extrema-, aunque tampoco admite la presencia "excesiva" de grasa. Cabe preguntarse por qué la imagen corporal femenina eficiente en el cumplimiento de las "funciones" de la construcción del cuerpo, actualmente, es de delgadez. La tendencia en nuestra sociedad a la lipofobia ha sido ya ampliamente documentada (Fischler 1990), también la mayor exigencia con las mujeres en este sentido, y se han elaborado teorías diversas para explicar esta situación (Gracia 2002: 365). Más allá de estas explicaciones sobre el porqué de la valoración de la delgadez —de indudable interés, por otro lado- interesa saber cómo afecta a la construcción del cuerpo en nuestra sociedad. Dependiendo de las vías por las que transite la construcción social del cuerpo, los individuos adoptarán unas $\mathrm{u}$ otras estrategias para modelar su imagen física. Entre estas estrate-

familia, y, por el otro, los cuidados afectuosos que se les prodigaba (Mahias 1985). En las ciudades italianas durante la Edad Media (Nahoum 1979, cit. en Contreras 1993), adquirir peso era un signo de riqueza y también de salud.

${ }^{2}$ Las religiones se han preocupado siempre por el cuerpo, para utilizarlo o para negarlo. La ascesis, que se fundamenta en la negación, enfatiza la renuncia a determinados alimentos o la restricción de su cantidad como una de las vías preferentes de su logro. El grado en el ayuno, en la renuncia o en la restricción se ha erigido en una forma de trazar la frontera entre los simples miembros de una comunidad religiosa y aquellos que llegan más lejos en el camino de la perfección espiritual o moral. 
gias se sitúan las que nos ocupan, las relacionadas con la alimentación, la actividad física y el deporte. Veamos pues cuáles son las connotaciones de la valoración de la delgadez en las mujeres, y de la ausencia de grasa, combinada con músculos visibilizados, en los hombres.

Entre los aspectos más negativos de la imagen corporal, en nuestras sociedades, destacan el sobrepeso y la obesidad. Como siempre cuando se trata de imagen corporal, la valoración o la desvalorización no hacen sólo referencia a una dimensión estética —que también — sino a otros aspectos de los individuos; a través de la imagen corporal se deducen rasgos de personalidad, carácter, moralidad, etc. En nuestra sociedad, se atribuyen una serie de características a la delgadez - y por tanto a los portadores de esta característica- y otras, a menudo opuestas, a la gordura. De una manera simplista, acaso reduccionista — en realidad las consideraciones son mucho más matizadas- pero útil a efectos analíticos, puede afirmarse que se atribuye a las personas delgadas una serie de cualidades, como ser ágil, activo, despierto, tener autocontrol, tener voluntad, etc., que se niegan, o se atribuyen sus contrarios, a las personas obesas y con sobrepeso.

¿Cómo interiorizan, interpretan y gestionan adolescentes y jóvenes las versiones actuales de la construcción del cuerpo? No resulta sorprendente que durante la adolescencia, periodo en el cual se modela la propia imagen, se sea particularmente sensible a la visión del otro que incide en la visión de uno mismo. La construcción del cuerpo adquiere, pues, en esta etapa particular relevancia. Además, como se ha dicho, no es sólo la identidad la que está en juego, también lo está la exhibición y la potenciación del atractivo sexual, y la importancia que éste adquiere durante la adolescencia y la juventud no se puede minimizar. La imagen corporal puede ser —no siempre lo es - un instrumento para conseguir pareja sexual, y tener éxito en ese sentido puede, en determinadas circunstancias, contribuir a satisfacer otras necesidades, tanto afectivas como materiales. Estas tres "funciones" de la construcción del cuerpo no son en absoluto banales, al contrario, y adquieren particular relevancia entre adolescentes y jóvenes. Esto supone optar por vías de construcción y movilizar estrategias que se van a relacionar estrechamente, a menudo, con la alimentación y la actividad física.

Por lo que respecta a la alimentación, numerosos estudios advierten del número creciente de adolescentes y de jóvenes con desajustes alimentarios porque "no se gustan a sí mismos"; es decir, no consiguen construir eficazmente su cuerpo, para proyectar, a través de la imagen corporal, la identidad aspirada, y eso a pesar de que, como advierten algunos autores (Bueno y Castro 1993), su peso y su talla encajen perfectamente dentro de parámetros considerados "normales". Desean ser más altos y estar más delgados. Resulta difícil, por no decir imposible, convencerlos de su "normali- 
dad" biológica, ya que la imagen deseada se rige por unos criterios y vehicula unos sentidos socialmente construidos y culturalmente interiorizados. Un gran número de adolescentes - muy en particular mujeres- se muestran intensamente preocupadas ante la posibilidad de estar gordas $-\mathrm{O}$ no delgadas - y esta preocupación afecta de distintas formas a su conducta alimentaria.

Las estrategias adoptadas para contener el peso dentro de los márgenes percibidos como aceptables suelen basarse en la omisión de comidas, la selección y la restricción. La primera conducta consiste en saltarse ingestas; lo más frecuente es dejar de desayunar y/o de merendar, en algunos casos se prescinde de la comida y la cena, sobre todo después de lo que se considera un sobreconsumo, para compensar, o antes, para prevenir. Si la tercera vía consiste básicamente en reducir o eliminar consumos, la primera se basa en sustituir productos considerados excesivamente calóricos - y por lo tanto contraproducentes para los objetivos propuestos- por sus equivalentes más "inofensivos", en particular los productos desnatados o los llamados light. En un estudio llevado a cabo entre adolescentes de Mallorca (Mójer, Benito y Tur 2001), se observa que los productos light son consumidos con frecuencia por éstos, pero con diferencias significativas entre chicos y chicas. Así, mientras que el $46 \%$ de las chicas mostraban una frecuencia de consumo diaria —más de tres veces al día- sólo el $31 \%$ de los chicos presentaba frecuencias similares. Se destaca también en este estudio el contraste existente entre la elevada ingesta de bebidas azucaradas, azúcar, dulces, bollería industrial y snacks, y el también alto consumo de productos light. Esta constatación evidencia, por un lado, el interés de estos adolescentes por construir su imagen corporal - y, por lo tanto, en nuestra sociedad, por la delgadez-, pero también su deseo de no renunciar a unos alimentos que para ellos son sinónimo de placer. De manera que el deseo de esbeltez no se traduce siempre, ni de manera mecánica, en restricción, si no que se buscan otras estrategias, entre ellas la actividad física, para conseguir un mismo objetivo.

En cualquier caso, lo que se evidencia con claridad es la voluntad de alcanzar un determinado peso, es decir, una determinada imagen, mediante la modificación de los comportamientos alimentarios. Esta situación ya había sido descrita en los años setenta, cuando Dwyer, Feldman y Mayer refirieron la elevada presencia de regímenes adelgazantes entre las mujeres, incluso entre aquellas consideradas delgadas. Pero lo que destaca, sobre todo, es la distancia existente entre la imagen valorada, en términos de delgadez, y la considerada deseable según los parámetros médicos. Esta insatisfacción de las adolescentes respecto a su imagen corporal aparece claramente en el estudio de Moses y otros (1989). En este estudio se re- 
partió un cuestionario en el que se interrogaba sobre el peso, las prácticas alimentarias y los conocimientos nutricionales a 326 adolescentes - mujeres- entre 13 y 18 años. Se documentó en esta ocasión una percepción distorsionada del peso corporal, en particular entre las chicas clasificadas como de peso insuficiente (un $36 \%$ del total). En cambio, aquellas que presentaban un peso adecuado o sobrepeso mostraban una percepción más acorde con su peso actual y la percepción del peso ideal según la talla. Pero es preciso destacar que la frecuencia de comportamientos considerados "anómalos" fue parecida en las tres categorías: muchas de las chicas encuestadas afirmaron realizar esfuerzos para restringir su ingesta y manifestaron temor a estar gordas, lo que equivale a no alcanzar los estándares de delgadez percibidos como apropiados. Alrededor de una tercera parte de las mujeres de peso insuficiente aparecían igualmente preocupadas, lo que evidenciaba que el extendido temor a la obesidad era independiente del peso. Otro estudio centrado en el grado de satisfacción con el peso corporal, figura, comportamientos alimentarios y prácticas relacionadas con el control del peso entre adolescentes es el de Moore (1988), para el que se repartieron cuestionarios anónimos a 854 adolescentes y mujeres jóvenes. El resultado fue que dos terceras partes de las encuestadas no estaban satisfechas con su peso actual, y que aproximadamente la mitad no lo estaba tampoco con su figura. Entre aquellas que se veían a sí mismas con sobrepeso, alrededor de un tercio deseaban una inadecuada —según los parámetros médicos- pérdida de peso. Muchas de las que deseaban perder una cantidad considerada excesiva presentaban en aquel momento un peso estimado como normal, algunas incluso insuficiente. También en este estudio aparecen casos de ayuno, vómitos autoprovocados y abuso de laxantes y diuréticos. Este desajuste entre peso "deseado" y peso "deseable" se refleja también en la encuesta realizada por la revista americana Glamour entre 33.000 mujeres, en la que el 75\% considera que su peso es excesivo, mientras que según las normas de la Metropolitan Life Insurance Company, solo el $25 \%$ presentaría un peso superior al deseable por altura y edad. Estudios más recientes llevados a cabo con adolescentes y mujeres jóvenes en España muestran la misma tendencia. Por ejemplo, el realizado sobre la percepción de la imagen corporal entre los estudiantes de la Universidad del País Vasco (Arroyo y otros 2003), en el que se analizaron las actitudes respecto al peso de 526 estudiantes, 158 hombres y 368 mujeres, muestra que las mujeres escogen figuras significativamente más delgadas que los hombres y manifiestan un grado de insatisfacción más elevado respecto a su imagen.

Lo que todos estos estudios muestran es que los parámetros médicos, que establecen criterios en términos de salud, no coinciden, o no lo hacen 
plenamente, con los parámetros socioculturales, que establecen los criterios en otros términos; en términos del lenguaje de la imagen corporal, culturalmente construidos y con sentidos y "funciones" sociales relevantes.

Hablar de construcción del cuerpo quiere decir modificarlo de alguna manera. Ciertas intervenciones pueden resultar muy agresivas e irreversibles, como la reducción de los pies de las mujeres chinas o los tatuajes, y otras pueden no incidir directamente y ser reversibles, como los tacones, para modificar la altura, o el uso de joyas, para embellecer o significar estatus. Sobre algunas dimensiones de la imagen corporal sólo se puede actuar -en principio- a través de la modificación de la apariencia. Este sería el caso de la altura; es difícil incrementar la altura realmente, pero es posible manipular su apariencia, por ejemplo mediante el uso de zapatos de tacón. Sobre otras, se puede actuar directamente, cambiando el cuerpo y no solo su apariencia. Para conseguir la imagen corporal deseada en relación a la delgadez, ausencia de grasa y visibilidad de músculos, se pueden adoptar estrategias que incidan o no directamente sobre el cuerpo, y que lo hagan de forma más o menos agresiva. Se puede utilizar ropa que disimule o oculte el exceso de grasa, o que acentúe la presencia de músculos, y estas serían prácticas no incidentes directamente sobre el cuerpo, puesto que no lo modifican, sino sólo su apariencia, o se puede operar mediante el control de la ingesta alimentaria —el llamado "control del peso" - y la práctica deportiva, es decir, actuando sobre el cuerpo de modo directo, modificándolo realmente. Estrategias que exigen una actuación consciente y constante, que requieren un esfuerzo y se caracterizan por considerarse dependientes íntegramente de la "voluntad" de los individuos. Así, no se considera que cambiar de altura o de facciones, por ejemplo, dependa de las decisiones de los individuos — aunque la cirugía estética esté modificando este escenario- pero sí se puede - y se debe- controlar el peso. Es esta noción de que existen estrategias efectivas para conseguir el objetivo, y que adoptarlas o no depende exclusivamente de las decisiones y las actuaciones, lo que conviene destacar. En relación al peso y el modelado de la figura, es fundamental la idea de autocontrol, y de ahí la centralidad del concepto de "control del peso".

VALORACIÓN DEL SOBREPESO Y DE LA OBESIDAD ENTRE LOS ADOLESCENTES Y LOS JÓVENES

La consideración social negativa del sobrepeso y la obesidad está plenamente interiorizada y es compartida por jóvenes y adolescentes (Espeitx y otros 2006). También aparece de manera generalizada la distinción entre sobrepeso y obesidad, y ésta no es sólo de grado, sino también de natura- 
leza. Mientras que la obesidad es percibida más a menudo como una enfermedad que el individuo padece, o que se puede explicar en parte por factores que no dependen exclusivamente de éste — predisposición genética, funcionamiento deficiente de la tiroides...-, el sobrepeso resultaría únicamente de los malos hábitos o de prácticas poco convenientes por parte de quien lo padece. Es decir, el sobrepeso es considerado como responsabilidad absoluta de el que lo "sufre", mientras que la obesidad estaría menos bajo el control del obeso ${ }^{3}$.

En cualquier caso, entre los jóvenes, la creencia de que es conveniente controlar los kilos, dentro de los límites socialmente aceptados, está ampliamente extendida, tanto entre hombres como entre mujeres. En los adolescentes, esta consideración se incrementa con la edad —es mayor a los 16-18 años que a los 12-13- y significativamente más generalizada entre las chicas que entre los chicos, aunque es una percepción extendida entre ambos sexos y en todas las edades. Ahora bien, esta creencia muy a menudo se matiza con observaciones como "unos kilos de más no tienen importancia", o "lo importante es sentirse bien con uno mismo, en lugar de estar pendientes de los modelos estéticos de moda". Esta relativización del sobrepeso, si no es muy pronunciado, aparece con mucha frecuencia, tanto entre chicas como entre chicos. Sin embargo, en general se aplica a los demás en mucha mayor medida a uno mismo. Es decir, es igual que el amigo o amiga pese algo más de lo considerado deseable, pero no da igual salirse uno mismo de los parámetros valorados. Aquello que se contempla con indulgencia en los demás puede generar un intenso malestar en uno mismo, lo que evidencia la complejidad de la percepción del cuerpo. Debe tenerse en cuenta, además, que los más indulgentes con el sobrepeso ajeno y que minimizan en mayor medida la necesidad de ejercer control sobre el peso, son aquellos que nunca han tenido ningún episodio de sobrepeso, real o percibido, y que nunca han considerado que mantenerse dentro de un determinado peso exigiera un esfuerzo consciente. Esta tendencia a quitarle importancia a la cuestión puede molestar, incluso indignar a aquellos que sí se creen obligados a una vigilancia constante, a veces mediante un "sacrificio" personal percibido como considerable ${ }^{4}$. En

3 "Yo creo que la obesidad y el sobrepeso son cosas muy distintas. La obesidad es una enfermedad, por que el cuerpo no quema, o acumula líquidos... o por hipotiroidismo... estas cosas. El sobrepeso es diferente, es por que comes más de lo que quemas, y ya está" (hombre, 26 años).

4 "Claro que tu no consideras importante controlar el peso... ¡Tu siempre has estado delgado! ¡Tu no tienes problema, comas lo que comas, siempre estás delgado!" (mujer, 23 años). 
cualquier caso, se suele desproblematizar el sobrepeso ajeno, pero no tanto el propio.

Resulta significativo que sean los chicos los que más a menudo tiendan a achacar al sedentarismo la causa del sobrepeso, mientras que las chicas suelen considerar los excesos alimentarios como los principales "culpables". De manera que, para los primeros, el mejor instrumento para vencer el sobrepeso, la estrategia más eficaz para modelar el cuerpo, sería la actividad física y el deporte, mientras que para ellas la estrategia preferente sería controlar — reducir - la ingesta alimentaria. Aunque ambos, hombres y mujeres, contemplan las dos opciones como convenientes y complementarias, la diferencia reside en el énfasis que se pone en una u otra. Debe tenerse en cuenta, que, aunque se consideran complementarias, también se establecen distinciones entre la estrategia del control de la ingesta y la del ejercicio físico. Se considera que las dos contribuyen a conseguir el cuerpo al que se aspira, un "buen cuerpo", pero las aportaciones de cada estrategia a este objetivo se perciben como distintas. El deporte, la actividad física, aporta "tonicidad", flexibilidad, musculación, en definitiva, modela el cuerpo, el control de la ingesta aporta delgadez, ausencia de grasa; la combinación de las dos estrategias proporciona el cuerpo deseado5.

La consideración de que el sobrepeso se explica - y se corrige- por un desequilibrio entre el consumo alimentario y el gasto energético, es mayoritariamente compartida, como se ha dicho antes. Por lo tanto, para evitar el sobrepeso, es preciso buscar el equilibrio entre estos dos factores. Pero no siempre, ni todo el mundo concede el mismo papel a la actividad física y a la alimentación. Para algunos el control de la ingesta es el instrumento principal, y la actividad física el subsidiario, o viceversa. Intervienen en este sentido las diferencias individuales, por un lado, y las de género, por el otro. Entre las diferencias individuales destacan la facilidad $-\mathrm{O}$ dificultad- percibida para mantener comportamientos alimentarios restrictivos, la atracción - o su contrario- por la actividad física y el deporte y los hábitos adquiridos, tanto en relación a la alimentación como a la actividad física. Por lo que respecta a las diferencias de género, si se considera la variación existente entre la imagen corporal femenina valorada — centrada en la delgadez - y la masculina valorada — centrada en la musculación-, se entiende fácilmente que las estrategias preferentes de unos y otras no sean las mismas. Para las primeras, la vía preferente será el control de la

5 "Hay que combinar la alimentación con el ejercicio. No basta con ir al gimnasio. Porque si vas al gimnasio y luego te hartas de comer cosas que no convienen... y si no comes pero tampoco haces ejercicio, estás delgado pero no tienes buen cuerpo" (hombre, 25 años). 
ingesta, para ellos, el incremento de la actividad física. Y esto tiene consecuencias, por que no es lo mismo construir el cuerpo mediante un incremento de la actividad física, que hacerlo mediante la reducción de la ingesta. Reducción que a veces puede ser importante —y excesiva y problemática- puesto que la delgadez valorada está fuera del alcance de la constitución de muchas mujeres.

\section{ACTIVIDAD FÍSICA, DEPORTE Y CONSTRUCCIÓN DEL CUERPO}

Se observa que un número significativo de adolescentes incrementa su actividad física o se inicia en la práctica del deporte por razones "de peso", es decir, por motivaciones relacionadas con la construcción del cuerpo. En algunos casos, porque se considera que se tienen unos kilos "de más", y se quiere incidir en este estado de cosas a través de la práctica deportiva. En otros, porque, aunque el peso que se tiene se considere correcto, se pretende también mejorar la imagen corporal ${ }^{6}$. Los motivos para hacerlo ya se han comentado anteriormente. Durante la adolescencia, sobre todo a medida que ésta avanza, es frecuente ir abandonando las actividades deportivas extraescolares, impulsadas en los colegios y por los padres. En algunos casos, esto se traduce en un incremento del sedentarismo, porque el anterior deporte extraescolar se convierte en más horas delante del ordenador o en reuniones con los amigos. En otros, se produce un desplazamiento hacia otras prácticas, elegidas por los mismos individuos y ya no por sus padres. Los deportes de equipo, como el fútbol sala o el básquet siguen siendo ampliamente practicados a estas edades, pero acudir al gimnasio se convierte en una de las nuevas prácticas más extendidas.

Al gimnasio se puede ir solo o en grupos de amigos, y también allí es posible hacer nuevas relaciones, y ésta es una baza a su favor cuando se trata de adolescentes, y en menor medida también de jóvenes. Aunque entre los motivos aducidos para asistir regularmente a un gimnasio se menciona el "estar en forma" y también "divertirse", parece claro que una de las "funciones" de los ejercicios realizados en el gimnasio es modelar el cuerpo. También en este contexto se detectan diferencias de género. Así, en general, las chicas se decantan por fortalecer los glúteos, y los chicos por muscular brazos y pectorales. Es frecuente que las mujeres, al iniciar un programa de fitness, adviertan que ellas no pretenden ni desean desarrollar

6 "Yo empecé a ir al gimnasio, no por que estuviera gorda, entonces estaba normal. Tenía un cuerpo normal, y yo quería tener un cuerpo que destacara. Ya que no destacaba por ser la más delgada, podía destacar siendo la que mejor cuerpo tenía" (mujer, 21 años). 
músculos, sino más bien reducir grasa corporal y endurecer determinadas partes del cuerpo, mientras que los adolescentes y los hombres jóvenes valoran en mayor medida un cuerpo más "fuerte". La satisfacción que proporciona obtener resultados visibles, es decir, que la imagen corporal se modifique, efectivamente, en la dirección deseada, se convierte en un estímulo para mantener, o aumentar, la frecuencia de las visitas al gimnasio. Así, la construcción del cuerpo puede ser un motor poderoso en la disminución del sedentarismo en buena parte de adolescentes y jóvenes.

\section{LA EXIGENCIA DE DELGADEZ EN EL DEPORTE}

Se ha comentado ampliamente la valoración en nuestra sociedad de la delgadez, del control del peso y el modelado del cuerpo mediante la alimentación y el ejercicio. Además de interiorizar e interpretar esta valoración socialmente compartida de una forma de modelar el cuerpo, el deportista comparte representaciones y condicionantes objetivos, propios del deporte en el que participa. Uno de estos condicionantes es el de mantener un determinado peso, considerado adecuado para conseguir unos resultados óptimos. Esto supone que la alimentación se convierte en un aliado fundamental en la búsqueda de un mejor rendimiento deportivo.

Esta necesidad de mantenerse dentro de unos límites de peso fijos y rígidos es vivida con acuciosidad por los deportistas, pero también por sus entrenadores, que suelen ser un factor de presión sobre los comportamientos alimentarios del deportista de primer orden. Esta presión ejercida por los entrenadores ha sido descrita ampliamente (Dosil y González 2003: 39), y se ha destacado que el control del peso puede convertirse en una preocupación central para los entrenadores, de manera que los datos que muestra la báscula acaban convirtiéndose en la manifestación visible de la dedicación del deportista al objetivo de mejorar su rendimiento. El entrenador ejerce, pues, una presión considerable, pero ésta no es siempre directa, sino que se realiza indirectamente, cuando el deportista tiene unas determinadas ideas sobre lo que desea el entrenador con respecto a su peso, realizando dietas estrictas o utilizando métodos inadecuados, con el objetivo de satisfacer sus supuestas expectativas (ibid.).

Ahora bien, no todas las disciplinas deportivas plantean las mismas exigencias en relación al peso. Determinados deportes son particularmente restrictivos, por lo que la presión se vuelve particularmente intensa. Para estas prácticas resulta esencial la delgadez, y es en estas disciplinas donde se producen más casos de los llamados trastornos del comportamiento alimentario, por lo cual se habla de "deportes de riesgo". El Comité Olímpico de los Estados Unidos (USOC 1998) divide estos deportes de riesgo 
en cuatro grupos: el primero estaría integrado por los deportes de estética, el segundo por aquellos en los que el peso es un elemento diferenciador entre una categoría u otra, el tercero reuniría a los que se practican en un gimnasio, y finalmente estarían los deportes de resistencia, que se benefician de un bajo peso para rendir mejor (Dosil y Rodríguez 2003: 24). En los llamados deportes de estética el aspecto físico de los participantes es fundamental, puesto que se incluye dentro de la puntuación de las pruebas por parte de los jueces. En el caso de las mujeres, las características exigidas son: delgadez acusada, piernas largas y fibrosas, poco pecho y caderas estrechas. A los hombres se les exige musculación y elasticidad. Pero lo que debe destacar es que en ambos casos, la alimentación y el control del peso están en el centro de las estrategias. En los deportes en los que el peso es un elemento diferenciador entre una categoría y otra, es frecuente la opción de adelgazar rápidamente para competir en una categoría por debajo de la que correspondería. Se pretende con ello ganar con mayor facilidad a contrarios, de por sí más ligeros. Por lo que respecta a las prácticas de los gimnasios, debe distinguirse entre dos tendencias principales. Por un lado, están aquellos, mayoritariamente hombres, interesados sobre todo en la musculación. Por el otro, aquellas — mayoritariamente mujeresque mediante el aeróbic, el estep o el fitness, centran la atención en el modelado del cuerpo, y, por lo tanto, en el peso. En los deportes de resistencia que exigen un bajo peso para obtener un mayor rendimiento, como las carreras de caballos, la exigencia consiste en pesar lo menos posible, puesto que cuanto más ligero sea el jockey, más ventaja tendrá el caballo.

Así pues, la consideración de que a mayor delgadez, mejor rendimiento, está plenamente interiorizada en un elevado número de deportistas, de diferentes disciplinas, tanto hombres como mujeres. Esto comporta la frecuente valoración, entre estos deportistas, de que el peso deseable es inferior o muy inferior a su peso real 7 , y se traduce en la adopción de prácticas alimentarias restrictivas, en algunos casos muy restrictivas, a menudo también acompañadas de períodos de sobreconsumo, compañeros frecuentes de la autorrestricción intensa. Según algunos autores, la incidencia de ciertos regímenes dietéticos agresivos y de restricciones alimentarias severas es relativamente alta entre los deportistas (Zunzunegui y Román 1995: 61). Efectivamente, existen estudios que muestran que los deportistas que prac-

7 Se ha estudiado esta relación entre peso ideal y peso real (Dosil y Rodríguez 2003: 29) en los practicantes de diferentes deportes, y se ha observado que el $52 \%$ de los judokas, por ejemplo, están descontentos con su peso real y consideran que deberían pesar menos. La gimnasia rítmica es la disciplina en la que mayor insatisfacción con el propio peso se muestra, siendo sólo un 10,5\% de las gimnastas las que muestran conformidad con el suyo. 
tican una disciplina individual, refieren un mayor control de su peso corporal que la población en general ( $70 \%$ versus $30 \%$ ) (ibid.: 58 ). Si consideramos, además, el grupo de deportistas de élite, destaca un nivel de control del peso corporal muy superior al de la población general y también al del resto de deportistas ( $74 \%$ versus $40 \%$ y $51 \%$ respectivamente). Esto a pesar de que la investigación médica muestra que no existe una evidencia clara sobre esta relación positiva entre delgadez y rendimiento (Dosil y Rodríguez 2003: 25).

LAS COMPLEJAS RELACIONES ENTRE IMAGEN CORPORAL, ACTIVIDAD FÍSICA Y ALIMENTACIÓN

La construcción del cuerpo, con todas sus "funciones" y todos sus sentidos, incide, como se ha visto, en los comportamientos alimentarios y en la práctica de actividad física. Así, entre delgadez, musculación, alimentación, actividad física y deporte se entretejen múltiples vínculos. Entre estos, destacan las que podemos llamar las relaciones positivas entre alimentación, actividad física y deporte, y construcción del cuerpo, por sus efectos y aquellas que se pueden definir como relaciones negativas entre alimentación, deporte y construcción del cuerpo, por sus consecuencias.

Se ha observado (Espeitx y otros 2006) que entre adolescentes y jóvenes el modelado del físico se puede convertir en un potente motor de "reforma alimentaria". Esto sucede sobre todo en individuos con sobrepeso, que deciden conformar un cuerpo más acorde con los parámetros vigentes. Para ello intentan incrementar los considerados más beneficiosos para sus objetivos (verduras, hortalizas, frutas, pescado, lácteos desnatados...) y eliminar o reducir los consumos percibidos como más "peligrosos" (bollería, snacks, dulces, helados, embutidos...). También actúan sobre las técnicas culinarias, escogiendo la cocción al vapor, los hervidos, el consumo en crudo, en detrimento de los fritos y de las salsas. De forma que la preocupación por la imagen corporal puede contribuir muy positivamente en los comportamientos alimentarios. Además, iniciar un régimen adelgazante,o "pensar" la propia alimentación en función del control del peso, va unido a menudo a un incremento de la actividad física. Por un lado, porque se percibe como una excelente medida complementaria, por otro, porque el ejercicio permite modelar el cuerpo de una forma que no consigue el simple adelgazamiento. Debe tenerse en cuenta, también, que una pérdida inicial de peso por reducción de la ingesta, se traduce en una sensación de mayor ligereza y agilidad, por lo cual la realización de ejercicio físico se hace más fácil y placentera. Y una mayor satisfacción con el cuerpo construido favorece la práctica de deportes que exigen mostrar el cuerpo. 
A su vez, cuando existe un interés claro por construir el cuerpo, y se decide por ello, como principal estrategia, aumentar la actividad física, se pueden desencadenar procesos paralelos, que afectan directamente a la alimentación, en el sentido antes indicado. Pueden contribuir a ello, con sus consejos, los monitores deportivos y los profesores de educación física. Por su parte, la práctica regular de un deporte supone, en muchos casos, una mayor preocupación por la alimentación, en la medida en que ésta puede afectar al rendimiento deportivo. También suele suponer el contacto, asimismo regular, con un entrenador, que puede contribuir, mediante sus indicaciones, a una mejora de los comportamientos alimentarios ${ }^{8}$. En cualquier caso, lo que se observa es que actividad física, práctica deportiva y control de la ingesta se retroalimentan mutuamente, generando un círculo virtuoso. Pueden, por ello, redundar muy positivamente en el bienestar físico y mental de los adolescentes y jóvenes, tanto si son deportistas como simples aficionados.

Pero la construcción del cuerpo a través del deporte y la alimentación no siempre tiene repercusiones positivas. Por un lado, se observa que la restricción alimentaria severa va unida a la práctica exacerbada de actividad física, en determinadas fases de trastornos del comportamiento alimentario, como la anorexia y la bulimia. La suma de la actividad física intensa y la reducción drástica de la ingesta, en el caso de la anorexia, permite una pérdida de peso más rápida y contribuye a que la espiral del trastorno se precipite. En el caso de la bulimia, la práctica de ejercicio físico puede suceder a las fases de consumo compulsivo, al utilizarse como una práctica compensatoria, por lo que se convierte en uno de los elementos constituyentes del círculo perverso de sobreconsumo y restricción. En este sentido, para la persona afectada, el ejercicio físico puede llegar a asimilarse a una de las dimensiones de su trastorno, y cargarse, por tanto, de connotaciones negativas. También, una tendencia a llevar al extremo determinadas prácticas para modificar la imagen corporal puede conducir a trastornos como la vigorexia, que, de igual manera, llevan aparejados patrones alimentarios problemáticos, como el consumo abusivo de preparados proteínicos.

Las relaciones negativas entre alimentación y actividad física pueden también circular en dirección contraria. Así, un consumo alimentario exce-

8 "Yo sí, yo pienso lo que como. Me preocupa comer bien. Sí, siempre ha sido así? A ver, yo creo que esto lo he adquirido en casa, pero después, al irme introduciendo en el mundillo este del deporte, pues me ha ido interesando más y más el tema de la alimentación. Sí, el hecho de hacer deporte ha hecho que me interesara más por la alimentación. Por ejemplo, si el domingo tengo competición, pues estoy tres o cuatro días que una de las comidas son hidratos de carbono, pasta o arroz" (hombre, 27 años). 
sivamente elevado, que desemboque en un incremento de peso, puede provocar una disminución de la actividad física. Por un lado, un cuerpo más pesado dificulta la práctica de ejercicio, por el otro, el deseo de ocultar el cuerpo, al percibirse gordo $-\mathrm{y}$ no corresponder a los parámetros socioculturalmente valorados-, comporta a menudo el abandono de actividades que suponen mostrarlo, como nadar o acudir al gimnasio. Se refuerza así una relación perversa entre aumento de peso y descenso de la actividad física, lo que a su vez conlleva un mayor incremento de peso. Se puede caer así en un círculo vicioso, que repercuta negativamente en el bienestar físico y mental de jóvenes y adolescentes.

Cuando se trata de practicar deportes de los considerados "de riesgo" las consecuencias pueden ser muy negativas. En este caso, se puede concluir que los deportistas, en particular los que se dedican a determinadas disciplinas, como consecuencia de esta acentuada preocupación por el peso, superior a la de la población en general, muestran una mayor propensión a padecer algún trastorno de los comportamientos alimentarios (Dosil y Rodríguez 2003: 33) y otros problemas de salud asociados. En efecto, las mujeres deportistas que entrenan intensamente y consumen dietas bajas en energía son un grupo de riesgo para padecer amenorrea (ausencia de menstruación). Se trata típicamente de gimnastas, bailarinas, corredoras y nadadoras que mantienen muy bajos contenidos de grasa corporal por razones estéticas y deportivas. También las mujeres deportistas son de alto riesgo en cuanto a la deficiencia de hierro, debido al frecuente bajo consumo y a un comprobado incremento de las pérdidas del mismo (Zunzunegui y Román 1995: 260).

\section{ALGUNAS REFLEXIONES FINALES}

Existen relaciones, a veces con efectos positivos, a veces con efectos negativos, entre actividad física, deporte, alimentación y construcción del cuerpo. Las consecuencias más problemáticas, sin embargo, se relacionan en mucha mayor medida con la estrategia alimentaria — que supone adelgazar mediante restricción de la ingesta- que con la de incrementar la actividad física. Se ha señalado que la primera es escogida más a menudo por las mujeres, mientras que la segunda es preferida por los hombres. Esto se explica porque los cuerpos valorados son distintos en función del género, y la delgadez se atribuye, como valor, en mayor medida a las mujeres. Para conseguir delgadez — las mujeres- es más eficiente la estrategia alimentaria, del mismo modo que para conseguir un cuerpo fuerte — los hombres- es más eficaz la de la actividad física. Pero encierra más riesgos para el bienestar físico y psicológico de adolescentes y jóvenes restringir la ingesta alimentaria 
que practicar algún deporte. Y comporta mayores beneficios la segunda. Una combinación hábil de las dos puede dar resultados muy positivos. En cualquier caso, la preocupación, o la voluntad, o el interés por construir el cuerpo no son problemáticos en sí mismos, pero pueden serlo algunas de sus manifestaciones, y que lo sean dependerá de cómo se configure, de qué formas adopte el cuerpo social y culturalmente valorado.

Debe tenerse en cuenta, también, que el hecho de practicar algún deporte o de realizar actividad física —así como el no hacerlo- no se explican exclusivamente por la voluntad de "construir el cuerpo". Intervienen otros criterios que pueden ser también muy poderosos. Por un lado, se puede practicar deporte por el mero placer que ello reporta y por los beneficios que se observan en el humor y el estado de ánimo. También el hecho de que el deporte sea, a menudo, una actividad de ocio que se realiza con compañeros, le confiere el atractivo de las prácticas en un contexto de amistad y diversión. Practicar algún deporte, o determinada actividad física, supone además proponerse retos y conseguirlos, alcanzar metas, con todo lo que ello supone de bienestar psicológico y mejora de la autopercepción. Tampoco deben interpretarse las elecciones alimentarias a partir de la imagen corporal como único criterio, en este caso intervienen muchos otros factores, como el placer, los efectos de los alimentos en el estado de ánimo, la comensalidad, los hábitos adquiridos.... Aún así, el papel de la "construcción del cuerpo" en la decisión de llevar a cabo alguna actividad deportiva y de modificar los comportamientos alimentarios no debe despreciarse, porque puede tener, y en muchas ocasiones tiene, un peso considerable en ambas decisiones.

\section{BIBLIOGRAFÍA CITADA}

Alberdi, I.; Escario y N. MATAs. 2000. Les dones joves a Espanya. Barcelona: Fundació la Caixa, Col·lecció Estudis Socials.

Arroyo, M.; A. M. Rocando; L. Ansótegui; et al. 2003. "Percepción de la imagen corporal en estudiantes de la Universidad del País Vasco", en II Jornadas de Antropología de la Alimentación, Nutrición y Salud. Bilbao. Eusko Ikaskuntza, Sociedad de Estudios Vascos (en prensa).

Bueno Campaña, M. y A. Martín Castro. 1993. "Adquisición de los hábitos alimentarios propios y su influencia en la dieta de los adolescentes". Nutrición Clínica 2, Año XIII, Vol. 13.

Dosil, J. y M. GonZÁLEz. 2003. "El contexto del deportista y los trastornos de alimentación”, en J. Dosil, Trastornos de Alimentación en el Deporte: 35-44. Sevilla: Wanceulen Editorial Deportiva.

- y M. RodríGuez. 2003. "El problema del peso en el deporte", en J. Dosil, Trastornos de Alimentación en el Deporte. Sevilla: Wanceulen Editorial Deportiva. 
Espeitx, E. 2003. "La alimentación como instrumento: restricciones alimentarias severas, consumos desmesurados y "dietas adelgazantes", en II Jornadas de Antropología de la Alimentación, Nutrición y Salud. Bilbao: Eusko Ikaskuntza, Sociedad de Estudios Vascos, (en prensa).

-; J. CÁceres; A. Puchal et al. 2006. L'Alimentació dels joves en procés d'emancipació. Barcelona: Observatori de la Joventut, Generalitat de Catalunya.

Fischer, C. 1995. El (H)omnívoro. Barcelona: Anagrama.

GraCiA, M. 2002. "Los trastornos alimentarios como trastornos culturales: la construcción social de la anorexia nerviosa”, en M. Gracia (ed.), Somos lo que comemos: 349-375. Barcelona: Ariel.

Leiva Ruiz, M. T. y J. A. Casajús Mallén. 2004. Cineantropometría. Condición física. Estilo de vida de los escolares aragoneses ( 7 a 12 años).

Mahias, M. C. 1985. Délivrance et convivialité. Le système culinaire des Jaina. París : Éditions de la Maison des Sciences del'Homme.

Mójer, M.; E. Benito y J. Tur. 2001. "Evaluación del consumo de alimentos en adolescentes escolarizados de Palma de Mallorca". Revista Española de Nutrición Comunitaria 7 (1-2): 7-17.

Moore, C. 1988. "Body Image and Eating Behavior in adolescent Girls". Am. Jdis. Child. 142: 1114-1118.

Moses, N.; B. Mansour-May y A. Filma Lifshitz. 1989. "Fear of Obesity Among Adolescent Girls". Pediatrics 3: 393-8.

ZunZunegui, J. L. y Román. 1995. "La alimentación como fenómeno sociocultural", en J. L. Zunzunegui, Comportamiento y hábitos alimentarios en jóvenes deportistas: 239261. Vitoria: Diputación Foral de Álava, Instituto de Deportes. 